\title{
Comparativa de resultados de rehabilitación energética para viviendas en función del grado de mejora
}

\section{Comparison of results retrofit energy for single-family housing in function of degree of upgrade}

\author{
$\underline{\text { A. Pérez Fargallo }}^{(*)}$, J. M. Calama Rodríguez ${ }^{(*)}$, V. Flores Alés ${ }^{(*)}$
}

\section{RESUMEN}

El objetivo del artículo es el estudio de viabilidad en mejoras de eficiencia energética a través del análisis del ahorro energético y de la inversión económica. Los resultados se han obtenido de un modelo estudiado con distintas disposiciones, sistemas constructivos e intervenciones, incluyendo dos opciones de rehabilitación respecto a las transmitancias establecidas en el Apéndice D del DB-HE Orden FOM/1635/2013, con la finalidad de generar distintas subcategorías de edificios. El análisis se ha basado en las demandas y consumos obtenidos a partir una herramienta creada en base a la UNE-ENISO-13.790:2011 y a la ASTM-E917:2013. Una vez obtenidos los resultados se ha realizado una comparativa energética y económica de resultados. Este trabajo evalúa aspectos económicos y ahorros en las mejoras de eficiencia energética para viviendas unifamiliares, comparando distintas alternativas en función de la situación de partida, con la finalidad profundizar en indicadores válidos para actuaciones de EE en edificaciones existentes.

Palabras clave: Rehabilitación; energía; eficiencia; costes; viabilidad.

\section{ABSTRACT}

The aim of the article is to study the energy efficiency improvements feasibility through the analysis of energy savings and economic investment. The results were obtained from a model studied with different orientations, building systems and interventions, including two retrofit options regarding thermal transmittance, specified in Appendix D of the DBHE Order FOM / 1635/2013, in order to generate different subcategories of buildings. The analysis was developed on demands and consumption obtained from a tool created based on the UNE-EN-ISO-1379O: 2011 and ASTM-E917: 2013. Once obtained the results it was made an energetic and economic results comparison. This paper evaluates economics and energy efficiency savings improvements, for dwellings, comparing various alternatives according to the initial situation, in order to deepen into valid indicators for energy efficiency actions in existing buildings.

Keywords: Retrofit; energy; efficiency; costs; viability.

(*) Centro de Investigación en Tecnologías de la Construcción - Universidad del Bío-Bío (Chile).

${ }^{(* *)}$ Universidad de Sevilla (España).

Persona de contacto/Corresponding author: aperezf@ubiobio.cl (A. Pérez Fargallo)

Cómo citar este artículo/Citation: Pérez Fargallo, A., Calama Rodríguez, J. M., Flores Alés, V. (2016). Comparativa de resultados de rehabilitación energética para viviendas en función del grado de mejora. Informes de la Construcción, 68(541): e134, doi: http://dx.doi. org/10.3989/ic.15.048.

Licencia / License: Salvo indicación contraria, todos los contenidos de la edición electrónica de Informes de la Construcción se distribuyen bajo una licencia de uso y distribución Creative Commons Reconocimiento no Comercial 3.o. España (cc-by-nc). 


\section{INTRODUCCIÓN}

La eficiencia energética en el sector residencial es uno de los objetivos prioritarios de la Unión Europea (1) (2). Se ha estimado alcanzar un ahorro energético del $27 \%$ en el conjunto de los edificios residenciales de los países comunitarios para el año 2020 a través de las Directivas promulgadas por la Comisión Europea (3) (4). El parque de viviendas español se encuentra entre los menos eficientes energéticamente de Europa en consecuencia, se plantean ayudas para incentivar el reacondicionamiento térmico de edificios residenciales (5).

La rehabilitación en el sector residencial destaca como un área potencial clave en la reducción del consumo energético en la mayoría de los países. Aumentar la eficiencia energética de dicho sector estudiando la relación que guarda el ahorro energético obtenido con el beneficio económico es un paso fundamental en el estímulo social para ese cambio de paradigma (6) (7).

El ahorro energético tiene como objetivo previsto la reducción del consumo de energía sin afectar a la productividad, calidad, etc. y sin producir, claro está, un impacto ambiental mayor que la situación inicial por el efecto rebote (8).

Los organismos públicos en España programan actuaciones para la promoción e incentivación de medidas de mejora de edificaciones e instalaciones existentes; pero se está dejando a un lado la relación que guarda el ahorro de energía final obtenido con el esfuerzo necesario para alcanzarlo (9). Resulta interesante disponer de recursos que ayuden en la toma de decisiones sobre la reducción de consumo en los procesos energéticos del sector residencial existente estableciendo niveles óptimos de rentabilidad tal y como establece el Reglamento Delegado (UE) n 244/2012 (10).

Los Documentos Básicos del Código Técnico de la Edificación son los encargados de definir en España los estándares mínimos de calidad en la construcción de viviendas, sin embargo, la aplicación directa de estos puede suponer una gran inversión en una reforma, incluso pudiendo hacer inviable el proyecto por el impacto económico o social que produzca (11), por ello es preciso evaluar la rentabilidad de los sistemas de $\mathrm{EE}$ de forma individual o por paquetes de medidas (12). El objetivo de la rehabilitación es mejorar las condiciones del edificio, tratando de ser coherentes con las posibilidades económicas, las características del mismo y las posibles barreras de incorporación que existan (13), es posible que dichas características sean difíciles de conciliar respecto a un edificio de nueva planta.

En principio parece claro que las soluciones van a estar condicionadas al caso de estudio (14). Debido a esto, resulta interesante realizar un estudio de casos concretos en los que se planteen soluciones realistas seleccionando un amplio número de sistemas que permitan obtener una base de datos que sirva de modelo para generar unos criterios generales incluyendo criterios económicos (15).

En España existen documentos técnicos, sin carácter reglamentario, con la finalidad de facilitar la aplicación y los estudios de eficiencia energética. Los documentos reconocidos pueden ser: programas informáticos de calificación de eficiencia energética de carácter prestacional, especificaciones y guías técnicas o comentarios sobre la aplicación técnico- administrativa de la certificación de eficiencia energética de carácter prescriptivo y/o cualquier documento que facilite la aplicación del procedimiento, siempre y cuando no se refieran a la utilización de un producto o sistema particular.

Dichos documentos se basan fundamentalmente en la evaluación de las emisiones anuales de $\mathrm{CO}_{2}$ indicador que es tenido en cuenta en el coste global de intervenciones de EE a nivel macroeconómico, sin embargo indicadores consumo anual de energía primaria o el coste de inversión en medidas de mejoramiento no son tenidos en cuenta (16) (17), factores que son relevantes para el coste global a nivel financiero en medidas de EE en edificios existentes a la hora de conocer qué posibilidades tiene un edificio para mejorar su comportamiento, así como a la hora de incentivar económicamente las actuaciones (10).

\section{OBJETIVOS}

La presente propuesta de investigación busca enlazar diferentes puntos de vista de un elemento común, la rehabilitación energética. El objetivo general es el estudio energético y económico para la mejora de la envolvente térmica de viviendas en la región sur de España, analizando mejoras sobre muros, techos, suelos y huecos desde puntos de vista técnicos, energéticos y económicos.

Se persigue generar indicadores económicos validos para la evaluación de mejoras en viviendas existentes en función de distintas casuísticas como el tipo de intervención (exterior o interior), tipos de sistemas constructivos, la relación con otras edificaciones (aislada, pareada o entre medianeras) y grado de mejoramiento («Básico» para alcázar los estándares del Apéndice D del DB-HE Orden FOM/1635/2013 y «Plus» para superarlos) (18).

Por último, se pretende la obtención de conocimientos económicos para la ejecución de mejoras energéticas aplicadas a las distintas edificaciones y el análisis del ahorro energético en base a las comparativas de demandas energéticas, consumos y coste del ciclo de vida del modelo inicial con el rehabilitado. Se debe tener en cuenta que una simulación puede diferir de la realidad construida por múltiples factores como la similitud del clima real con los modelos climáticos oficiales, valores de temperatura de consigna, diferencias entre la envolvente térmica real y el modelo energético, existencia de entradas de aire incontroladas, divergencia entre la eficiencia teórica y real de los equipos de climatización, sistemas de ventilación manual, inadecuada elección de los equipos de climatización, ocupación y hábitos de los usuarios. Por todo ello, los valores obtenidos en la investigación son una estimación económica.

\section{METOdOLOGía}

El primer paso ha sido la selección de una serie de sistemas constructivos adecuados para la rehabilitación energética de huecos, fachadas y cubiertas. La elección de dichos sistemas se realizó a partir de la facilidad de ejecución, la rapidez y calidad de la misma y los materiales empleados. Una vez seleccionados los sistemas constructivos se procedió al cálculo de los costes que supondría la implementación de mejoras en la envolvente térmica tanto en el interior como en el exterior, el precio obtenido es un precio unitario funcional, el cual es el coste por unidad de un elemento constructivo formado por 

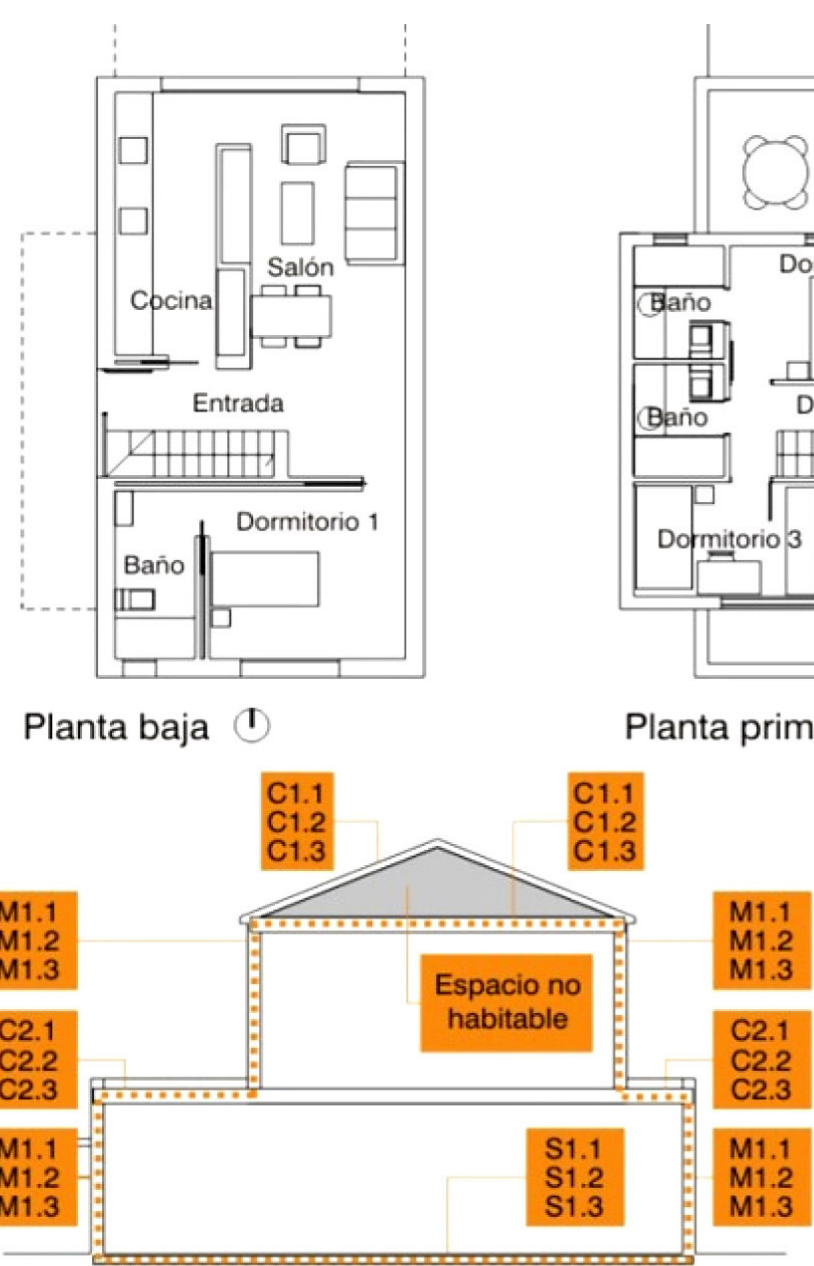

Sección longitudinal

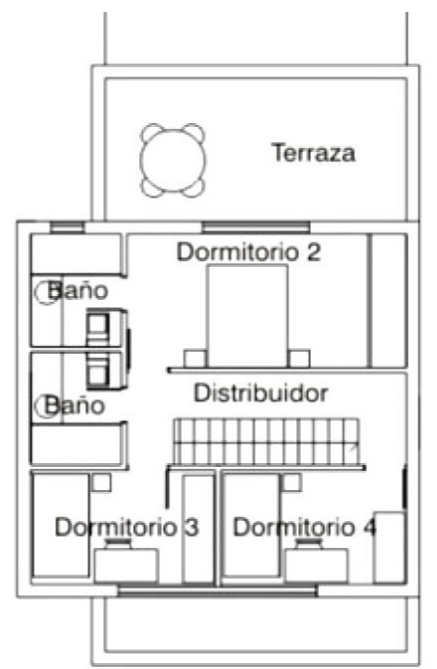

Planta primera

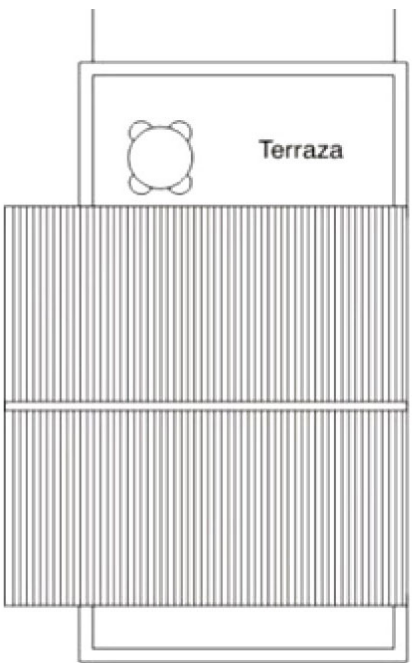

Cubierta

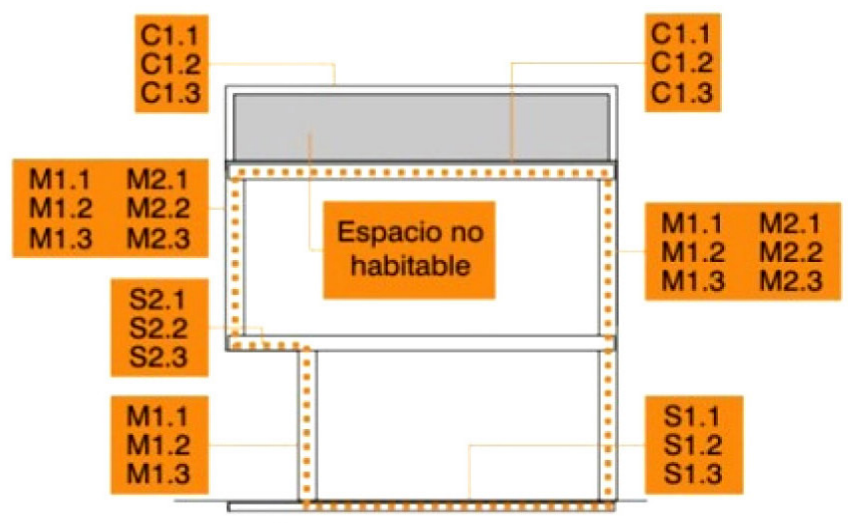

Sección transversal $\because \underbrace{0} \quad \begin{array}{lll}1 & 2 & 3\end{array}$

Figura 1. Modelo de vivienda usada para el estudio y claves de cerramientos para interpretación de la Tabla 2.

una combinación de elementos básicos, auxiliares y unitarios que constituyen un conjunto constructivo con una función compleja dentro de la obra (19).

Los sistemas constructivos del estado inicial de las edificaciones se determinaron a partir del análisis de la antigüedad de las viviendas a rehabilitar, así se dividieron en tres grupos: anterior al Real Decreto 2429/1979, de 6 de Julio, Normativa Básica de la edificación sobre Condiciones Térmicas de los Edificios (CT-79) con estructura de fábrica, anterior al CT-79 con estructura de hormigón y posterior al CT-79 con estructura de hormigón (ver Tabla 1). El uso de sistemas estructurales masivos (Muros de fábrica) frente al uso de sistemas estructurales de hormigón hace que se modifique el comportamiento de los cerramientos sin atender a la entraba en vigor del CT-79. Dicha clasificación atiente a las recomendaciones de subcategorización del Reglamento Delegado (UE) no 244/2012 el cual índica en su apartado 3 «Los diferentes parques de edificios pueden exigir una categorización diferente. En un país, la diferenciación basada en los materiales de construcción puede resultar la más adecuada, mientras que en otro la diferenciación puede basarse en la edad del edificio» (10) (11).

Con la intención de generar parámetros cuantificables en función de la edad, del tipo de intervención, características constructivas y tipología, se seleccionó un edificio mode- lo situado en la ciudad de Sevilla (España) (Zona climática B4), el cual se simuló con diferentes disposiciones y sistemas constructivos, creándose así nueve subcategorías (ver Tabla 1). Se fijaron características comunes: zona climática B4, uso, orientación, superficies de la envolvente, volumen, número de plantas; para de esta forma localizar diferencias y similitudes entre las rehabilitaciones de las edificaciones construidas con distintos sistemas constructivos, estructurales y tipologías. En cuanto al tipo de intervención, se tuvo en cuenta su ejecución tanto interior como exterior, por lo que las 9 subcategorías de la Tabla 1, pasaron a ser 18. El edificio cuenta con planta baja y primera, cubierta a dos aguas y terrazas, orientado en la dirección $\mathrm{N}-\mathrm{S}$, tiene una superficie útil de $103,90 \mathrm{~m}^{2}$ (ver Figura 1). En la Tabla 1 se determina la superficie de huecos para cada una de las fachadas del modelo objeto de estudio (puertas y ventanas).

Con dichos modelos se evaluó la disminución en la demanda energética de las mejoras propuestas en los distintos submodelos a partir de una herramienta generada en base a la UNE-EN ISO 13.790:2011 (20) y se realizó el análisis de los costes económicos para la rehabilitación de la mejora de la envolvente a partir de la ASTM E918 2013 (21).

Los costes de mejoramientos en la envolvente se calcularon por metro cuadrado de superficie de edificio a partir de solu- 
Tabla 1. Superficies de huecos (puertas y ventanas) para cada una de las fachadas del modelo y subcategorías objeto de estudio.

\begin{tabular}{|c|c|c|}
\hline Orientación & $\begin{array}{c}\text { Superficie total } \\
\left(\mathbf{m}^{2}\right)\end{array}$ & $\begin{array}{c}\text { Superficie } \\
\text { huecos }\left(\mathrm{m}^{2}\right)\end{array}$ \\
\hline Norte & 43,21 & 11,99 \\
\hline Este & 55,34 & 0,00 \\
\hline Oeste & 55,34 & 4,20 \\
\hline Sur & 40,21 & 4,95 \\
\hline \multicolumn{3}{|c|}{ Subcategorías objeto de estudio } \\
\hline $\begin{array}{c}\text { Tipología } \\
\text { edificatoria }\end{array}$ & Normativa & Tipo de estructura \\
\hline Aislada & Anterior al CT-79 & $\begin{array}{l}\text { Estructura de } \\
\text { fábrica }\end{array}$ \\
\hline Aislada & Anterior al CT-79 & $\begin{array}{l}\text { Estructura de } \\
\text { hormigón }\end{array}$ \\
\hline Aislada & Posterior al CT-79 & $\begin{array}{l}\text { Estructura de } \\
\text { hormigón }\end{array}$ \\
\hline Pareada & Anterior al CT-79 & $\begin{array}{c}\text { Estructura de } \\
\text { fábrica }\end{array}$ \\
\hline Pareada & Anterior al CT-79 & $\begin{array}{c}\text { Estructura de } \\
\text { hormigón }\end{array}$ \\
\hline Pareada & Posterior al CT-79 & $\begin{array}{c}\text { Estructura de } \\
\text { hormigón }\end{array}$ \\
\hline Entre medianeras & Anterior al CT-79 & $\begin{array}{l}\text { Estructura de } \\
\text { fábrica }\end{array}$ \\
\hline Entre medianeras & Anterior al CT-79 & $\begin{array}{l}\text { Estructura de } \\
\text { hormigón }\end{array}$ \\
\hline Entre medianeras & Posterior al CT-79 & $\begin{array}{l}\text { Estructura de } \\
\text { hormigón }\end{array}$ \\
\hline
\end{tabular}

*CT-79 Real Decreto 2429/1979, de 6 de Julio. Normativa Básica de la edificación sobre Condiciones Térmicas de los Edificios.

ciones tipo preestablecidas, concluyendo dicho análisis con unos ratios económicos que se espera faciliten la toma de decisiones para obras de rehabilitación energética de una forma estimativa en función del año de construcción, su tipología y tipo de intervención.

El aislamiento seleccionado para la rehabilitación de las diferentes partes que componen la envolvente ha sido el poliestireno extruido (XPS), su elección se basó en la gran versatilidad que ofrece a la hora de su colocación en distintas disposiciones. Dicho material será usado en muros, suelos y cubiertas, de forma que sean comparables los datos obtenidos del análisis entre la rehabilitación de cada uno de ellos. El espesor del aislante dependerá del valor de transmitancias ha obtener para alcanzar los estándares de transmitancia del Apéndice D del DB-HE Orden FOM/1635/2013 (18) (ver Tabla 2).

Para la rehabilitación de fachadas se seleccionaron dos tipos de terminación en función del tipo de intervención; actuación exterior, enfoscado de cemento con malla y pintura blanca y actuación interior, guarnecido de yeso con malla y pintura blanca.

Para la rehabilitación de suelos, al igual que para las fachadas, se han elegido dos tipos de actuaciones. En el caso del suelo apoyado sobre el terreno, sólo se considerará la intervención por el interior, puesto que no es viable la intervención exterior. Los tipos de intervención seleccionadas en función del tipo de actuación, son los siguientes; actuación exterior, reposición del material existente en el tipo de cerramiento elegido previo a la intervención (enfoscado de mortero o falso techo de escayola); actuación interior, reposición sobre el material existente (en los suelos se ha considerado un solado cerámico tomado con mortero).

Para la intervención en cubiertas se tuvo en cuenta la tipología (plana o inclinada). Para la actuación exterior en cubiertas planas se consideró la ejecución del aislamiento y la nueva solería sobre la solería existente, mientras que en cubiertas inclinadas se consideró la demolición con recuperación del material para aplicar el aislamiento y tras ello reponer el acabado de tejas cerámicas. En las actuaciones interiores en ambos tipos de cubierta se eligió la opción de reponer el material de acabado que se había considerado en el cerramiento, en el caso de cielo raso se sustituirá por falso techo de escayola o en el caso de guarnecido de yeso se aplicaría el mismo acabado sobre el aislamiento.

La elección de los sistemas de rehabilitación de carpinterías se basó en las carpinterías iniciales consideradas para los modelos de estudio. En el caso de viviendas con estructura de fábrica previas al CT-79 cuya carpintería inicial se ha considerado de madera, la rehabilitación de sus huecos de realizaría con carpinterías de madera y vidrio en cámara 6/8/5. Las carpinterías de madera se han considerado color marrón con las relaciones marco/vidrio $25 \%-75 \%$ y $50 \%-50 \%$ en función del tamaño del hueco. En el resto de los modelos se incluyo el mismo tipo de vidrio, sustituyendo las carpinterías en función de la orientación; para Sur y Oeste se uso marco de aluminio y en los huecos orientados a Norte marco de aluminio con rotura de puente térmico debido (RPT) a la mayor limitación de transmitancias para tal orientación. Las carpinterías de aluminio se han considerado color gris con las relaciones marco/vidrio $15 \%-85 \%$ y $30 \%-70 \%$ en función del tamaño del hueco (ver Tabla 2). Los factores solares modificados cumplen con los requerimientos del apartado D2.8 del apéndice D del DB HE para cada orientación y porcentaje de huecos.

Tras la evaluación de los 18 modelos con rehabilitación básica se realizará una serie de mejoras en las actuaciones con la intención de sobredimensionar los aislamientos mínimos para alcanzar la rehabilitación «plus». Las mejoras para el estudio «plus» consistirán básicamente en un incremento del espesor de aislamiento de $3 \mathrm{~cm}$. en función del aislamiento básico y una mejora en los sistemas de huecos considerando carpinterías de PVC de dos cámaras y vidrios con cámara 6/8/5 bajo emisivo.

Todos los datos anteriores fueron incorporados en una herramienta de cálculo que evalúa cada uno de los factores anteriormente descritos. La finalidad de dicha herramienta fue la de simplificar el procedimiento. Para el predimensionado de climatización y calefacción dicha herramienta usa el método de cálculo mensual de la norma UNE EN ISO 13790:2011 (20) basado en el balance de pérdidas y ganancias útiles para el régimen de invierno, así como para el régimen de verano; a partir de la demanda obtenida por dicho procedimiento y los rendimientos de los sistemas de climatización así como del precio de las energías empleadas se han obtenido los consumos para realizar el análisis de rentabilidad de las inversiones en base a la ASTM E917-05 2013 (21).

Para el cálculo de demandas de climatización se han considerado 1,2 renovaciones de aire hora en el edificio sin rehabilitar y $0,75 \mathrm{R} / \mathrm{h}$ con el edificio rehabilitado, unas temperaturas de consigna de $19{ }^{\circ} \mathrm{C}$ para invierno y $26{ }^{\circ} \mathrm{C}$ para verano durante todas las horas del día y un valor de $2,1 \mathrm{~W} / \mathrm{m}^{2}$ para las ganan- 
Tabla 2. Parámetros característicos de la envolvente para cada subcategoría de estudio.

\begin{tabular}{|c|c|c|c|c|c|c|c|c|}
\hline \multirow{2}{*}{ 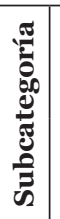 } & \multirow{2}{*}{\multicolumn{3}{|c|}{$\begin{array}{l}\text { Elemento por } \\
\text { subcategoría } \\
\text { (Ver Figura 1) }\end{array}$}} & \multirow{2}{*}{$\begin{array}{c}\text { Transmitancia } \\
\text { inicial } \\
\left(\mathbf{W} / \mathbf{m}^{2} \mathbf{K}\right)\end{array}$} & \multicolumn{2}{|c|}{$\begin{array}{c}\text { Transmitancia rehabilitación básica } \\
\text { (W/m²K) / Espesor de aislamiento } \\
\text { (XPS) - Carpintería vidrio }\end{array}$} & \multicolumn{2}{|c|}{$\begin{array}{l}\text { Transmitancia rehabilitación plus } \\
\text { (W/m²) / Espesor de aislamiento } \\
\text { (XPS) - Carpintería vidrio }\end{array}$} \\
\hline & & & & & Inter. Exterior & Inter. Interior & Inter. Exterior & Inter. Exterior \\
\hline \multirow{11}{*}{ 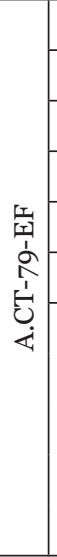 } & & & M1.1 & 1,26 & $0,72 / 2 \mathrm{cms}$ & $0,72 / 2 \mathrm{cms}$ & $0,39 / 6 \mathrm{cms}$ & $0,39 / 6 \mathrm{cms}$ \\
\hline & & & M1.1 & 1,91 & $0,71 / 3 \mathrm{cms}$ & $0,71 / 3 \mathrm{cms}$ & 0,44 / $6 \mathrm{cms}$ & $0,44 / 6 \mathrm{cms}$ \\
\hline & & & C1.1 & 1,80 & $0,42 / 4 \mathrm{cms}$ & $0,41 / 6 \mathrm{cms}$ & $0,23 / 8 \mathrm{cms}$ & $0,30 / 10 \mathrm{cms}$ \\
\hline & & & 1 (Terraza) & 1,54 & $0,41 / 6 \mathrm{cms}$ & $0,41 / 6 \mathrm{cms}$ & $0,33 / 8 \mathrm{cms}$ & $0,28 / 10 \mathrm{cms}$ \\
\hline & & & S1.1 & 0,66 & \multicolumn{2}{|c|}{$0,51 / 8 \mathrm{cms}$} & \multicolumn{2}{|c|}{$0,51 / 8 \mathrm{cms}$} \\
\hline & \multicolumn{3}{|c|}{ S2.1 (Aire) } & 1,23 & $0,50 / 4 \mathrm{cms}$ & $0,50 / 4 \mathrm{cms}$ & $0,32 / 8 \mathrm{cms}$ & $0,32 / 8 \mathrm{cms}$ \\
\hline & \multirow{5}{*}{ 总 } & \multirow{2}{*}{$\mathrm{S}$} & (50 \% marco) & 4,10 & \multicolumn{2}{|c|}{ 4,10 Sin intervención } & \multicolumn{2}{|c|}{ 4,10 Sin intervención } \\
\hline & & & (25\% marco) & 4,80 & \multicolumn{2}{|c|}{ 4,80 Sin intervención } & \multicolumn{2}{|c|}{ 4,8o Sin intervención } \\
\hline & & $\mathrm{O}$ & (25\% marco) & 4,80 & \multicolumn{2}{|c|}{ 4,80 Sin intervención } & \multicolumn{2}{|c|}{ 4,8o Sin intervención } \\
\hline & & \multirow{2}{*}{$\mathrm{N}$} & (25\% marco) & 4,80 & \multicolumn{2}{|c|}{ 2,65 / Madera (6/8/5) } & \multicolumn{2}{|c|}{ 2,25 / PVC $2 \mathrm{C}(6 / 8 / 5)$ Low E. } \\
\hline & & & $(50 \%$ marco $)$ & 4,10 & \multicolumn{2}{|c|}{ 2,8o / Madera (6/8/5) } & \multicolumn{2}{|c|}{ 2,30 / PVC $2 \mathrm{C}(6 / 8 / 5)$ Low E. } \\
\hline \multirow{11}{*}{ 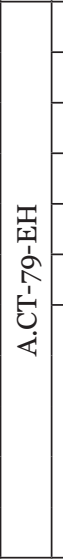 } & \multicolumn{3}{|r|}{ M1.2 } & 1,36 & $0,76 / 2 \mathrm{cms}$ & $0,76 / 2 \mathrm{cms}$ & $0,40 / 6 \mathrm{cms}$ & $0,40 / 6 \mathrm{cms}$ \\
\hline & \multicolumn{3}{|r|}{ M2.2 } & 1,40 & $0,77 / 2 \mathrm{cms}$ & $0,77 / 2 \mathrm{cms}$ & $0,40 / 6 \mathrm{cms}$ & $0,40 / 6 \mathrm{cms}$ \\
\hline & \multicolumn{3}{|r|}{$\mathrm{C} 1.2$} & 1,54 & $0,44 / 3 \mathrm{cms}$ & $0,42 / 6 \mathrm{cms}$ & $0,21 / 8 \mathrm{cms}$ & $0,28 / 10 \mathrm{cms}$ \\
\hline & \multicolumn{3}{|r|}{$\mathrm{C} 2.2$} & 1,77 & $0,43 / 6 \mathrm{cms}$ & $0,43 / 6 \mathrm{cms}$ & $0,34 / 8 \mathrm{cms}$ & $0,29 / 10 \mathrm{cms}$ \\
\hline & & & S1.2 & 0,66 & 0,51 & $\mathrm{cms}$ & 0,51 & $\mathrm{cms}$ \\
\hline & & & S2.2 & 1,31 & $0,52 / 4 \mathrm{cms}$ & $0,52 / 4 \mathrm{cms}$ & $0,27 / 10 \mathrm{cms}$ & $0,27 / 10 \mathrm{cms}$ \\
\hline & & $S$ & (30 \% marco) & 5,70 & $3,80 / \mathrm{Al}$ & $(6 / 8 / 5)$ & 2,30 / $\mathrm{PVC} 2 \mathrm{C}$ & /8/5) Low E. \\
\hline & $n$ & $\mathrm{~S}$ & (15\% marco) & 5,70 & $3,49 / \mathrm{Al}$ & $(6 / 8 / 5)$ & $2,25 / \mathrm{PVC} 2 \mathrm{C}$ & /8/5) Low E. \\
\hline & $\underset{\mathscr{d}}{\Xi}$ & $\mathrm{O}$ & (15\% marco) & 5,70 & $3,49 / \mathrm{Al}$ & $(6 / 8 / 5)$ & $2,25 / \mathrm{PVC} 2 \mathrm{C}$ & /8/5) Low E. \\
\hline & 牙 & $N$ & $(15 \%$ marco $)$ & 5,70 & $3,30 / \mathrm{R}$ & $(6 / 8 / 5)$ & $2,25 / \mathrm{PVC} 2 \mathrm{C}$ & $/ 8 / 5)$ Low E. \\
\hline & & IN & (10 \% marco) & 5,70 & $3,20 / \mathrm{R}$ & $(6 / 8 / 5)$ & $2,30 / \mathrm{PVC}_{2} \mathrm{C}$ & /8/5) Low E. \\
\hline & & & M1.3 & 0,69 & $0,49 / 2 \mathrm{cms}$ & $0,49 / 2 \mathrm{cms}$ & $0,22 / 6 \mathrm{cms}$ & $0,22 / 6 \mathrm{cms}$ \\
\hline & & & M2.3 & 0,70 & $0,70 /-$ & $0,70 /-$ & $0,30 / 4 \mathrm{cms}$ & $0,30 / 4 \mathrm{cms}$ \\
\hline & & & C1.3 & 1,01 & $0,48 / 3 \mathrm{cms}$ & $0,48 / 3 \mathrm{cms}$ & $0,16 / 6 \mathrm{cms}$ & $0,20 / 8 \mathrm{cms}$ \\
\hline & & & C2.3 & 1,05 & $0,39 / 2 \mathrm{cms}$ & $0,36 / 4 \mathrm{cms}$ & $0,27 / 6 \mathrm{cms}$ & $0,27 / 6 \mathrm{cms}$ \\
\hline 空 & & & S1.3 & 0,66 & 0,51 & $\mathrm{cms}$ & 0,51 & $\mathrm{cms}$ \\
\hline $\begin{array}{c}2 \\
1 \\
1 \\
1\end{array}$ & & & S2.3 & 1,31 & $0,52 / 4 \mathrm{cms}$ & $0,52 / 4 \mathrm{cms}$ & $0,27 / 10 \mathrm{cms}$ & $0,27 / 10 \mathrm{cms}$ \\
\hline$\ddot{\varphi}$ & & & (30 \% marco) & 5,70 & $3,80 / \mathrm{Al}$ & $(6 / 8 / 5)$ & $2,30 / \mathrm{PVC}_{2} \mathrm{C}$ & /8/5) Low E. \\
\hline & in & $\mathrm{S}$ & (15\% marco) & 5,70 & $3,49 / \mathrm{Al}$ & $(6 / 8 / 5)$ & $2,25 / \mathrm{PVC} 2 \mathrm{C}$ & /8/5) Low E. \\
\hline & $\underset{\mathscr{E}}{\Xi}$ & $\mathrm{O}$ & (15\% marco) & 5,70 & $3,49 / \mathrm{Al}$ & $(6 / 8 / 5)$ & $2,25 / \mathrm{PVC} 2 \mathrm{C}$ & /8/5) Low E. \\
\hline & 武 & N & (15\% marco) & 5,70 & $3,30 / \mathrm{R}$ & $(6 / 8 / 5)$ & $2,25 / \mathrm{PVC} 2 \mathrm{C}$ & /8/5) Low E. \\
\hline & & N & (10 \% marco) & 5,70 & $3,20 / \mathrm{R}$ & $(6 / 8 / 5)$ & $2,30 / \mathrm{PVC}_{2} \mathrm{C}$ & /8/5) Low E. \\
\hline
\end{tabular}

cias internas. Se ha considerado un factor de protección de las persianas con la finalidad de evitar las ganancias solares en verano. Los valores de puentes térmicos se han obtenido del DA DB-HE/3 (22).

La obtención de los costes globales de cada una de las subcategorías no incluye aquellos costes que se suponen similares en todas ellas (Costos de mantenimiento, energía para iluminación, ventilación y ACS y costos de explotación), teniéndose en cuenta la inversión en medidas de EE y los consumos energéticos para climatización, considerándose un valor residual similar. El periodo de estudio analizado es de 40 años, la tasa de actualización del 3 \% y para el cálculo de los factores de actualización para cada período se ha considerado un interés de un $4 \%$. Los factores de conversión de energía primaria a energía final son 2,492 MWhte.p./MWhe.f. para electricidad y 2,355 MWhte.p./MWhe.f. para gas natural, los coeficientes de paso de energía final a emisiones de $\mathrm{CO}_{2}$ son 0,34 y $0,5 \mathrm{tCO}_{2} / \mathrm{MWhe}$.f respectivamente (23). Los rendimientos teóricos tenidos en cuenta para la obtención del consumo de climatización han sido $75 \%$ para el sistema de calefacción y 190 \% para el de refrigeración. Los valores para el cálculo de la evolución de los precios de las energías han sido obtenidos de Eurostat Stadistics Explainted (24). 
Una vez obtenidos los resultados se realizará una comparativa energética y económica de las 36 subcategorías analizadas teniendo en cuenta el consumo teórico del edificio sin contemplar criterios de confort adaptativo o incapacidad de climatizar la vivienda por parte del usuario (25).

\section{RESULTADOS Y DISCUSIÓN}

En el primer paso, se analizarán las estimaciones de ahorro obtenidos en función del tipo de intervención (exterior o interior), sistemas constructivos, la relación con otras edificaciones (aislada, pareada o entre medianeras) y el grado de mejoramiento. En la Tabla 3 se presentan las diferencias de ahorro energético, económico y de reducción de emisiones de $\mathrm{CO}_{2}$ que se pueden obtener según el alcance de la rehabilitación energética.

La menor reducción en demanda tras la intervención se produce en las viviendas entre medianeras ejecutadas según el CT-79 con actuación exterior (41,22\%), siendo la mayor para el mismo tipo de vivienda anterior al CT-79 con estructura de fábrica (54,05\%). La diferencia, si comparamos entre edificaciones con similares tipologías, se encuentra entre un 9,37 y un 12,83 \% para la actuación básica. En la rehabilitación «plus» las diferencias se encuentran entre un 4,92-7,01\%, siendo la construcción aislada A.CT-79-EH-E la que ofrece un mejor comportamiento y la vivienda entre medianeras D.CT-79-EH-I la que en este caso produce una menor reducción. De lo anterior se deduce que a un mayor nivel de mejoramiento menor será la influencia de los sistemas constructivos iniciales, teniendo un mejor comportamiento en un nivel mayor de rehabilitación los edificios aislados a diferencia de lo que ocurría en un nivel básico. Las correlaciones anteriores son aplicables a las disminuciones de consumos, ahorros económicos y reducciones de emisiones de $\mathrm{CO}_{2}$.

Como se puede observar en la Tabla 3, no existen diferencias sustanciales con respecto al tipo de actuación, exterior e interior, o en función al tipo de vivienda; la diferencia se encuentra marcada fundamentalmente por la normativa de ejecución o sistemas constructivos previos; pero la influencia del tipo de actuación se incrementa con la opción «plus». Las diferencias entre ambas actuaciones radican en la intervención que se realiza en la cubierta inclinada puesto que los valores de transmitancias son diferentes en función de la posición del aislante con respecto a la cubierta y en la mejor corrección de puentes térmicos desde el exterior.

La rehabilitación plus incrementa desde un 11,79 a un $20,96 \%$ el ahorro en demanda energética, siendo la vivienda que ofrece una mayor diferencia con respecto la mejora básica la pareada D.CT-79-EH y la menor la vivienda entre medianeras A.CT-79-EH, dicha afirmación se justifica en el estado inicial de las construcciones, las edificaciones ejecutadas aplicando el CT-79 tienen un mejor comportamiento inicial y en consecuencia una menor reducción de demanda cuando se rehabilita hasta un estándar básico, sin embargo tal diferencia se incrementa cuando el mejoramiento es mucho mayor.

Si en la intervención tan sólo se evaluaran conceptos energéticos, sería lógico decantarse por la opción que mayor ahorro supone al modelo de estudio; pero para un análisis completo se debe analizar el incremento de coste que supone esa op-

Tabla 3. Porcentaje de reducciones y ahorro en función del grado de mejoramiento.

\begin{tabular}{|c|c|c|c|c|c|c|c|c|c|c|}
\hline \multirow{2}{*}{\multicolumn{2}{|c|}{ Subcategoría }} & \multirow{3}{*}{ 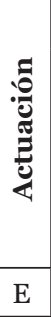 } & \multicolumn{4}{|c|}{ Rehabilitación Básica } & \multicolumn{4}{|c|}{ Rehabilitación Plus } \\
\hline & & & 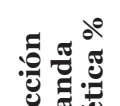 & ฮ̊ @ & $\begin{array}{r}d e \\
0 \\
0 \\
0\end{array}$ & 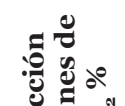 & : & ฮ & 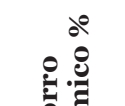 & 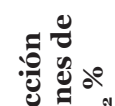 \\
\hline \multirow{6}{*}{ 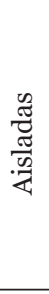 } & \multirow{2}{*}{ A.CT-79-EF } & & $50,53 \%$ & $49,14 \%$ & $50,77 \%$ & $50,93 \%$ & $64,86 \%$ & $62,70 \%$ & $65,23 \%$ & $65,47 \%$ \\
\hline & & $\mathrm{I}$ & $50,34 \%$ & $48,98 \%$ & $50,58 \%$ & $50,73 \%$ & $64,63 \%$ & $62,50 \%$ & $64,99 \%$ & $65,23 \%$ \\
\hline & \multirow{2}{*}{ A.CT-79-EH } & $\mathrm{E}$ & $52,34 \%$ & $53,07 \%$ & $52,21 \%$ & $52,13 \%$ & $68,56 \%$ & $68,13 \%$ & $68,64 \%$ & $68,68 \%$ \\
\hline & & $\mathrm{I}$ & $52,40 \%$ & $53,12 \%$ & $52,28 \%$ & $52,20 \%$ & $68,17 \%$ & $67,79 \%$ & $68,23 \%$ & $68,27 \%$ \\
\hline & \multirow{2}{*}{ D.CT-79-EH } & $\mathrm{E}$ & $42,97 \%$ & $44,66 \%$ & $42,67 \%$ & $42,47 \%$ & $63,64 \%$ & $63,34 \%$ & $63,70 \%$ & $63,73 \%$ \\
\hline & & $\mathrm{I}$ & $43,00 \%$ & $44,68 \%$ & $42,70 \%$ & $42,50 \%$ & $63,11 \%$ & $62,89 \%$ & $63,15 \%$ & $63,17 \%$ \\
\hline \multirow{6}{*}{ 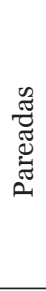 } & \multirow{2}{*}{ A.CT-79-EF } & $\mathrm{E}$ & $52,93 \%$ & $51,89 \%$ & $53,12 \%$ & $53,24 \%$ & $65,63 \%$ & $63,98 \%$ & $65,92 \%$ & $66,12 \%$ \\
\hline & & $\mathrm{I}$ & $52,74 \%$ & $51,74 \%$ & $52,92 \%$ & $53,04 \%$ & $65,39 \%$ & $63,79 \%$ & $65,68 \%$ & $65,88 \%$ \\
\hline & \multirow{2}{*}{ A.CT-79-EH } & $\mathrm{E}$ & $52,86 \%$ & $53,40 \%$ & $52,76 \%$ & $52,70 \%$ & $68,39 \%$ & $68,07 \%$ & $68,45 \%$ & $68,49 \%$ \\
\hline & & $\mathrm{I}$ & $52,92 \%$ & $53,45 \%$ & $52,83 \%$ & $52,77 \%$ & $67,98 \%$ & $67,72 \%$ & $68,02 \%$ & $68,05 \%$ \\
\hline & \multirow{2}{*}{ D.CT-79-EH } & $\mathrm{E}$ & $41,68 \%$ & $42,68 \%$ & $41,50 \%$ & $41,38 \%$ & $62,64 \%$ & $62,36 \%$ & $62,70 \%$ & $62,73 \%$ \\
\hline & & $\mathrm{I}$ & $41,71 \%$ & $42,70 \%$ & $41,53 \%$ & $41,41 \%$ & $62,08 \%$ & $61,91 \%$ & $62,12 \%$ & $62,14 \%$ \\
\hline \multirow{6}{*}{ 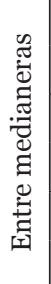 } & \multirow{2}{*}{ A.CT-79-EF } & $\mathrm{E}$ & $54,05 \%$ & $53,02 \%$ & $54,24 \%$ & $54,37 \%$ & $65,89 \%$ & $64,44 \%$ & $66,15 \%$ & $63,33 \%$ \\
\hline & & $\mathrm{I}$ & $53,86 \%$ & $52,86 \%$ & $54,04 \%$ & $54,16 \%$ & $65,65 \%$ & $64,25 \%$ & $65,91 \%$ & $66,08 \%$ \\
\hline & \multirow{2}{*}{ A.CT-79-EH } & $\mathrm{E}$ & $53,19 \%$ & $53,59 \%$ & $53,12 \%$ & $53,07 \%$ & $68,27 \%$ & $68,02 \%$ & $68,32 \%$ & $68,35 \%$ \\
\hline & & $\mathrm{I}$ & $53,26 \%$ & $53,64 \%$ & $53,19 \%$ & $53,14 \%$ & $67,84 \%$ & $67,66 \%$ & $67,87 \%$ & $67,89 \%$ \\
\hline & \multirow{2}{*}{ D.CT-79-EH } & $\mathrm{E}$ & $41,22 \%$ & $41,92 \%$ & $41,10 \%$ & $41,10 \%$ & $62,13 \%$ & $61,91 \%$ & $62,16 \%$ & $62,19 \%$ \\
\hline & & I & $41,25 \%$ & $41,94 \%$ & $41,12 \%$ & $41,04 \%$ & $61,55 \%$ & $61,45 \%$ & $61,57 \%$ & $61,58 \%$ \\
\hline
\end{tabular}


ción mejorada con respecto a la opción básica, así como su periodo de retorno.

Es preciso indicar que el coste, a diferencia del ahorro energético, sí se verá muy influenciado por las condiciones de partida, se debe aclarar que el coste exterior en viviendas pareadas o entre medianeras contempla costes de rehabilitación interior en muros, debido a que la medianería es imposible rehabilitarla por el exterior, al igual que sucede con los suelos en contacto con el terreno.

La mayor inversión en rehabilitación de muros en la opción básica habría que realizarla en un edificio aislado A.CT-79-EH con actuación exterior y la menor con tres veces menos de in-

Tabla 4. Estimación de costes para la rehabilitación energética para viviendas en función del grado de rehabilitación (básico o plus).

\begin{tabular}{|c|c|c|c|c|c|c|c|c|}
\hline \multirow{2}{*}{\multicolumn{2}{|c|}{ Subcategorías }} & \multirow{2}{*}{ 氖 } & \multicolumn{3}{|c|}{ Rehabilitación Básica } & \multicolumn{3}{|c|}{ Rehabilitación Plus } \\
\hline & & & Aislamiento & Acabados & Total & Aislamiento & Acabados & Total \\
\hline \multicolumn{9}{|c|}{ Costes de rehabilitación Muros } \\
\hline \multirow{6}{*}{$\begin{array}{l}\frac{\pi}{\pi} \\
\frac{\pi}{\pi} \\
\frac{\pi}{4} \\
\frac{\pi}{4}\end{array}$} & \multirow{2}{*}{ A.CT-79-EF } & $\mathrm{E}$ & $3.182,76 €$ & $3.647,37 €$ & $6.830,13 €$ & $4.702,32 €$ & $3.647,37 €$ & $8.349,69 €$ \\
\hline & & I & $1.840,86 €$ & $2.248,84 €$ & $4.089,70 €$ & $3.366,09 €$ & $2.248,84 €$ & $5.614,96 €$ \\
\hline & \multirow{2}{*}{ A.CT-79-EH } & $\mathrm{E}$ & $3.182,76 €$ & $3.648,04 €$ & $6.830,80 €$ & $4.702,32 €$ & $3.648,04 €$ & $8.350,36 €$ \\
\hline & & $\mathrm{I}$ & $1.530,90 €$ & $2.226,66 €$ & $3.757,56 €$ & $3.056,13 €$ & $2.226,66 €$ & $5.282,79 €$ \\
\hline & \multirow{2}{*}{ D.CT-79-EH } & $\mathrm{E}$ & $3.182,76 €$ & $3.648,04 €$ & $6.830,80 €$ & $4.702,32 €$ & $3.648,04 €$ & $8.350,36 €$ \\
\hline & & $\mathrm{I}$ & $1.530,90 €$ & $2.226,66 €$ & $3 \cdot 757,56 €$ & $3.056,13 €$ & $2.226,66 €$ & $5.282,79 €$ \\
\hline \multirow{6}{*}{ 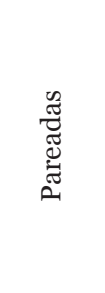 } & \multirow{2}{*}{ A.CT-79-EF } & $\mathrm{E}$ & $3.293,99 €$ & $3.234,53 €$ & $6.528,53 €$ & $4.702,32 €$ & $3.234,53 €$ & $7 \cdot 936,85 €$ \\
\hline & & I & $1.952,65 €$ & $2.248,84 €$ & $4.201,49 €$ & $3.366,09 €$ & $2.248,84 €$ & $5.614,93 €$ \\
\hline & \multirow{2}{*}{ A.CT-79-EH } & $\mathrm{E}$ & $3.182,76 €$ & $3.235,21 €$ & $6.417,97 €$ & $4.702,32 €$ & $3.235,21 €$ & $7 \cdot 937,53 €$ \\
\hline & & $\mathrm{I}$ & $1.530,90 €$ & $2.226,66 €$ & $3 \cdot 757,56 €$ & $3.056,13 €$ & $2.226,66 €$ & $5.282,79 €$ \\
\hline & \multirow{2}{*}{ D.CT-79-EH } & $\mathrm{E}$ & $2.250,83 €$ & $2.571,68 €$ & $4.822,51 €$ & $4.479,85 €$ & $3.235,21 €$ & $7.715,06 €$ \\
\hline & & I & $1.082,65 €$ & $1.563,14 €$ & $2.645,78 €$ & $2.832,56 €$ & $2.226,66 €$ & $5.059,22 €$ \\
\hline \multirow{6}{*}{ 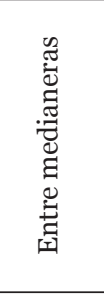 } & \multirow{2}{*}{ A.CT-79-EF } & $\mathrm{E}$ & $3.337,87 €$ & $3.071,68 €$ & $6.409,55 €$ & $4.702,32 €$ & $3.071,68 €$ & $7.774,00 €$ \\
\hline & & I & $1.996,74 €$ & $1.987,10 €$ & $3.983,85 €$ & $3.366,09 €$ & $2.248,84 €$ & $5.614,93 €$ \\
\hline & \multirow{2}{*}{ A.CT-79-EH } & $\mathrm{E}$ & $3.182,76 €$ & $3.072,35 €$ & $6.255,11 €$ & $4.702,32 €$ & $3.072,35 €$ & $7 \cdot 774,67 €$ \\
\hline & & $\mathrm{I}$ & $1.530,90 €$ & $2.226,66 €$ & $3 \cdot 757,56 €$ & $3.056,13 €$ & $2.226,66 €$ & $5.282,79 €$ \\
\hline & \multirow{2}{*}{ D.CT-79-EH } & $\mathrm{E}$ & $1.883,22 €$ & $3.072,35 €$ & $4.955,57 €$ & $4.392,10 €$ & $3.072,35 €$ & $7.464,45 €$ \\
\hline & & $\mathrm{I}$ & $905,82 €$ & $1.301,39 €$ & $2.207,22 €$ & $2.744,36 €$ & $2.226,66 €$ & $4.971,03 €$ \\
\hline \multicolumn{9}{|c|}{ Costes de rehabilitación Suelos } \\
\hline \multirow{6}{*}{ 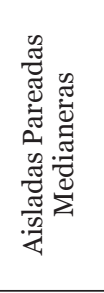 } & \multirow{2}{*}{ A.CT-79-EF } & $\mathrm{E}$ & $689,63 €$ & $1.453,95 €$ & $2.143,59 €$ & $829,64 €$ & $1.453,95 €$ & $2.283,59 €$ \\
\hline & & $\mathrm{I}$ & $1.328,95 €$ & $1.444,86 €$ & $2.773,80 €$ & $1.645,52 €$ & $1.444,86 €$ & $3.090,38 €$ \\
\hline & \multirow{2}{*}{ A.CT-79-EH } & $\mathrm{E}$ & $692,69 €$ & $1.347,41 €$ & $2.040,10 €$ & $840,14 €$ & $1.347,41 €$ & $2.187,55 €$ \\
\hline & & I & $1.346,90 €$ & $1.444,86 €$ & $2.791,76 €$ & $1.681,35 €$ & $1.444,86 €$ & $3.126,21 €$ \\
\hline & \multirow{2}{*}{ D.CT-79-EH } & $\mathrm{E}$ & $692,69 €$ & $1.347,41 €$ & $2.040,10 €$ & $840,14 €$ & $1.347,41 €$ & $2.187,55 €$ \\
\hline & & $\mathrm{I}$ & $1.346,90 €$ & $1.444,86 €$ & $2.791,76 €$ & $1.681,35 €$ & $1.444,86 €$ & $3.126,21 €$ \\
\hline \multicolumn{9}{|c|}{ Costes de rehabilitación Cubiertas } \\
\hline \multirow{6}{*}{ 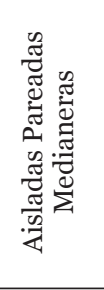 } & A CT-70-EF & $\mathrm{E}$ & $962,19 €$ & $2467,24 €$ & $3429,43 €$ & $1478,88 €$ & $2467,24 €$ & $3946,12 €$ \\
\hline & 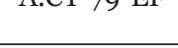 & $\mathrm{I}$ & $685,96 €$ & $1757,13 €$ & $2443,09 €$ & $846,06 €$ & $1757,13 €$ & $2603,19 €$ \\
\hline & A CT-70-FH & $\mathrm{E}$ & $856,39 €$ & $2467,24 €$ & $3323,64 €$ & $1478,88 €$ & $2467,24 €$ & $3946,12 €$ \\
\hline & R.C1-/9-Li1 & $\mathrm{I}$ & $1167,52 €$ & $1012,41 €$ & $2179,93 €$ & $1634,55 €$ & $1012,41 €$ & $2646,60 €$ \\
\hline & $\mathrm{D} C \mathrm{~T}_{-} \mathrm{s}$ & $\mathrm{E}$ & $650,76 €$ & $2467,24 €$ & $3118,00 €$ & $1167,52 €$ & $2467,24 €$ & $3634,76 €$ \\
\hline & D. C1-79-EII & $\mathrm{I}$ & $553,60 €$ & $1012,41 €$ & $1566,01 €$ & $714,86 €$ & $1012,41 €$ & $1727,27 €$ \\
\hline & & & & Costes de r & ilitación $\mathbf{H}$ & & & \\
\hline & A.CT-79-EF & $\mathrm{E}$ & & $1497,80 €$ & & & $3201,94 €$ & \\
\hline 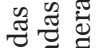 & & $\mathrm{I}$ & & & & & & \\
\hline 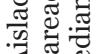 & A.CT-79-EH & $\mathrm{E}$ & & $3450,28 €$ & & & $570146 €$ & \\
\hline & & $\mathrm{I}$ & & & & & & \\
\hline & D.CT-79-EH & $\mathrm{E}$ & & $3450,28 €$ & & & $5701,46 €$ & \\
\hline
\end{tabular}


versión en la vivienda entre medianeras D.CT-79-EH con actuación interior, de forma análoga sucede con el mejoramiento plus, a salvedad de que la diferencia entre ambas inversiones es la mitad. El incremento de precio para pasar de una rehabilitación básica a plus en muros se encuentra entre 21-125 \%.

En la Tabla 4 se observa que existen diferencias de costes sustanciales en función de la tipología (Aislada D.CT-79-EH-E y entre medianeras D.CT-79-EH-E), si bien tales diferencias son menores cuanto mayor es el nivel de mejoramiento pasándose de una diferencia del $37 \%$ al $12 \%$, o en el caso de la actuación interior para los mismos modelos, del $70 \%$ al $6 \%$. Se puede afirmar que cuanto mejor sea la actuación menor influencia tendrá la tipología en las diferencias de inversión. Algo similar ocurre con los sistemas constructivos o normativa con la cual se ha ejecutado el edificio (Pareada A.CT79-EF-I y D.CT-79-EH-I), en la mejora básica la diferencia de costes entre la aplicación o no del CT-79 puede suponer un $59 \%$ de incremento entre esos dos edificios, sin embargo en la opción plus esa diferencia es de un $11 \%$.

El valor de aislamiento para el nivel básico se encuentra entre un 38-52 \% de la inversión y entre un 55-60 \% para la opción plus. Los costes obtenidos de las actuaciones desde el exterior son entre un $55 \%$ y un $124 \%$ mayores que en intervenciones interiores para un mejoramiento básico; y entre un $38 \%$ y un $58 \%$ en la opción plus, lo cual indica que cuando mayor sea el grado de mejoramiento menor será la influencia en la inversión del tipo de actuación (Exterior-Interior).

Analizando la Tabla 4 se deduce que la máxima y la mínima inversión para mejoras en suelos hay que realizarla en los casos en los que las viviendas con EH (Estructura de Hormigón), la máxima cuando la rehabilitación se realice desde el interior y la mínima cuando sea exterior, existiendo una diferencia entre ambas de un $27 \%$ en rehabilitación básica y un $30 \%$ en rehabilitación plus. Con respecto al incremento de costes entre un mejoramiento básico o plus para este tipo de cerramiento es preciso indicar que se encuentra entre un 6,5 y $12 \%$.

A diferencia de los muros, en los suelos no existen diferencias de costes sustanciales en función de la tipología, a su vez no existe una discrepancia relevante de costes en función de los sistemas o normativas de ejecución (o,6-5 \%) puesto que la mayoría de las viviendas construidas con aplicación del CT79 carece de aislamiento en suelos, no afectando de forma apreciable el nivel de mejoramiento a dichas aseveraciones.

El valor de aislamiento para el nivel básico se encuentra entre un 32-48 \% de la inversión y entre un 36-54 \% para la opción plus. Las diferencias de inversión en la opción básica a la hora de decantarse por el tipo de actuación pueden encontrarse entre un 23-27 \% y en la opción «plus» entre 26-30 \%, resultando más económicas las aplicadas en el exterior, observándose que a mayor grado de mejoramiento mayor influencia en la inversión del tipo de actuación (exterior-interior).

En la Tabla 4 se puede observar que no existen diferencias de costes en función de la tipología, produciéndose la mayor inversión en rehabilitación de cubiertas en la opción básica en un edificio A.CT-79-EH con actuación exterior y la menor con un $54 \%$ menos de inversión en la vivienda D.CT-79-EH con actuación interior. De igual forma ocurre con el mejoramiento «plus», encontrándose la diferencia entre ambas viviendas en torno a un $56 \%$. El sobrecoste para alcanzar el mejoramiento «plus» se encuentra entre 6,5-21,5\%.
Existen diferencias sustanciales en función del tipo de actuación, tales diferencias son mayores cuanto mayor es el nivel de rehabilitación, pasándose de una diferencia del $40 \%$ a $99 \%$ para la opción básica a un rango de 49-110 \% para la opción «plus»; a mejor actuación mayor influencia tendrá el tipo de actuación. De forma análoga ocurre con los sistemas constructivos o normativa con la cual se ha ejecutado el edificio (Aislada A.CT-79-EF-I y D.CT-79-EH-I), en la mejora básica la diferencia de costes entre la aplicación o no del CT-79 puede suponer un $36 \%$ más de inversión, y opción plus esa diferencia es de un $34 \%$.

El valor de aislamiento para el nivel básico se encuentra entre un 21-54 \% de la inversión y entre un 32-61 \% para la opción «plus».

Las carpinterías de aluminio con rotura de puente térmico y vidrio con cámara 6/8/5 son más económicas que las carpinterías de PVC con vidrio 6/8/5 bajo emisivo. Así resulta que la opción básica es entre un 39 y un 53 \% menor que la opción plus. El coste de las carpinterías tal y como se puede ver en la Tabla 4 no se encuentran influenciados por el tipo de actuación o la tipología edificatoria.

En la vivienda A.CT-79-EF se ha supuesto carpintería de madera, la cual ofrece menores transmitancias térmicas que el sistema de aluminio. Así, para alcanzar un nivel básico de rehabilitación no es necesaria la mejora de todos los huecos, sustituyéndose únicamente en los huecos orientados a norte, dicho criterio se ha seguido en la rehabilitación plus, suponiendo dicha situación un ahorro en la inversión de entre un 43 y un $56 \%$.

En la Tabla 5 realiza un análisis del porcentaje de inversión por elemento con respecto a la inversión total que habría que realizar en cada uno de los modelos estudiados para ambas opciones, además del retorno de la inversión. A partir de un análisis global de resultados se puede considerar que para la opción básica el mayor porcentaje de inversión se tendría que hacer en los cerramientos verticales opacos de una vivienda aislada A-CT-79-EF con actuación exterior al igual que en el caso plus, y el menor en el caso de rehabilitación básica en ese mismo modelo para la unidad de carpinterías; sin embargo, en un mejoramiento plus la menor inversión se la llevaría el mejoramiento de suelos en una vivienda A.CT-79-EH con actuación exterior.

De forma pormenorizada se puede ver que el reparto de porcentajes se encuentra relacionado con el tipo de intervención (exterior o interior), la normativa con la cual se ejecutó/sistemas constructivos, la relación con otras edificaciones (aislada, pareada o entre medianeras) y el grado de mejoramiento. En la opción de rehabilitación básica el mayor peso en inversión lo poseen los muros, a salvedad de las viviendas pareadas o entre medianeras D.CT-79-EH con intervención interior en las cuales el mayor coste de rehabilitación lo tendrían las carpinterías. Para esta misma opción de mejoramiento las menores inversiones habría que realizarlas en cubiertas, suelos y carpinterías; diferenciando en función del tipo de actuación, exterior-suelos, interior-cubiertas o al sistema constructivo inicial de las carpinterías, en el cual en caso de ser de madera sería la partida con un porcentaje inferior.

En la rehabilitación «plus» el mayor peso en inversión es para muros en la mayoría de los casos y en las carpinterías para intervenciones interiores en viviendas A.CT-79-EH y 
Tabla 5. Estimación de porcentajes de inversión por elemento con respecto a la inversión total y pay-back en función del grado de rehabilitación (básico o plus).

\begin{tabular}{|c|c|c|c|c|c|c|c|c|c|c|c|c|}
\hline \multirow{2}{*}{\multicolumn{2}{|c|}{ Subcategorías }} & \multirow{3}{*}{. } & \multicolumn{5}{|c|}{ Rehabilitación Básica } & \multicolumn{5}{|c|}{ Rehabilitación Plus } \\
\hline & & & \multirow{2}{*}{$\begin{array}{l}\text { Muros } \\
49,13 \% \\
\end{array}$} & \multirow{2}{*}{$\begin{array}{l}\text { Suelos } \\
15,42 \% \\
\end{array}$} & \multirow{2}{*}{$\begin{array}{c}\text { Cubiertas } \\
24,67 \%\end{array}$} & \multirow{2}{*}{$\begin{array}{l}\text { Carpint. } \\
10,77 \%\end{array}$} & \multirow{2}{*}{$\begin{array}{c}\begin{array}{c}\text { Retorno } \\
\text { Pay- } \\
\text { back } \\
\text { (Años) }\end{array} \\
13,23\end{array}$} & \multirow{2}{*}{$\begin{array}{l}\text { Muros } \\
46,96 \%\end{array}$} & \multirow{2}{*}{$\begin{array}{l}\text { Suelos } \\
12,84 \%\end{array}$} & \multirow{2}{*}{$\begin{array}{c}\text { Cubiertas } \\
22,19 \%\end{array}$} & \multirow{2}{*}{$\begin{array}{l}\text { Carpint. } \\
18,01 \%\end{array}$} & \multirow{2}{*}{$\begin{array}{c}\begin{array}{c}\text { Retorno } \\
\text { Pay- } \\
\text { back } \\
\text { (Años) }\end{array} \\
13,17\end{array}$} \\
\hline \multirow{6}{*}{$\begin{array}{l}\frac{n}{0} \\
\frac{\pi}{0} \\
\frac{\pi}{4} \\
\frac{\pi}{4}\end{array}$} & & & & & & & & & & & & \\
\hline & & I & $37,85 \%$ & $25,67 \%$ & $22,61 \%$ & $13,86 \%$ & 10,32 & $38,70 \%$ & $21,30 \%$ & $17,94 \%$ & $22,07 \%$ & 10,78 \\
\hline & \multirow{2}{*}{ A.CT-79-EH } & $\mathrm{E}$ & $43,84 \%$ & $13,09 \%$ & $21,33 \%$ & $21,74 \%$ & 13,72 & $41,37 \%$ & $10,84 \%$ & $19,55 \%$ & $28,25 \%$ & 13,52 \\
\hline & & $\mathrm{I}$ & $31,01 \%$ & $23,04 \%$ & $17,99 \%$ & $27,95 \%$ & 10,65 & $31,53 \%$ & $18,66 \%$ & $15,79 \%$ & $34,02 \%$ & 11,29 \\
\hline & \multirow{2}{*}{ D.CT-79-EH } & $\mathrm{E}$ & $44,24 \%$ & $13,21 \%$ & $20,20 \%$ & $22,35 \%$ & 24,10 & $42,02 \%$ & $11,01 \%$ & $18,29 \%$ & $28,69 \%$ & 20,78 \\
\hline & & I & $32,49 \%$ & $24,14 \%$ & $13,54 \%$ & $29,83 \%$ & 18,04 & $33,36 \%$ & $19,74 \%$ & $10,91 \%$ & $36,00 \%$ & 16,70 \\
\hline \multirow{6}{*}{ 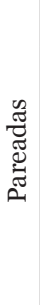 } & \multirow{2}{*}{ A.CT-79-EF } & $\mathrm{E}$ & $48,01 \%$ & $15,76 \%$ & $25,22 \%$ & $11,01 \%$ & 12,75 & $45,70 \%$ & $13,15 \%$ & $22,72 \%$ & $18,44 \%$ & 13,12 \\
\hline & & I & $38,49 \%$ & $25,41 \%$ & $22,38 \%$ & $13,72 \%$ & 10,27 & $38,70 \%$ & $21,30 \%$ & $17,94 \%$ & $22,07 \%$ & 11,00 \\
\hline & \multirow{2}{*}{ A.CT-79-EH } & $\mathrm{E}$ & $42,31 \%$ & $13,45 \%$ & $21,91 \%$ & $22,33 \%$ & 14,17 & $40,14 \%$ & $11,06 \%$ & $19,96 \%$ & $28,84 \%$ & 14,24 \\
\hline & & I & $31,01 \%$ & $23,04 \%$ & $17,99 \%$ & $27,95 \%$ & 11,31 & $31,53 \%$ & $18,66 \%$ & $15,80 \%$ & $34,02 \%$ & 12,14 \\
\hline & \multirow{2}{*}{ D.CT-79-EH } & $\mathrm{E}$ & $35,91 \%$ & $15,19 \%$ & $23,22 \%$ & $25,69 \%$ & 22,77 & $40,10 \%$ & $11,37 \%$ & $18,89 \%$ & $29,64 \%$ & 21,59 \\
\hline & & I & $25,31 \%$ & $26,71 \%$ & $14,98 \%$ & $33,00 \%$ & 17,71 & $32,40 \%$ & $20,02 \%$ & $11,06 \%$ & $36,51 \%$ & 17,69 \\
\hline \multirow{6}{*}{ 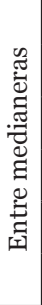 } & \multirow{2}{*}{ A.CT-79-EF } & $\mathrm{E}$ & $47,55 \%$ & $15,90 \%$ & $25,44 \%$ & $11,11 \%$ & 12,70 & $45,18 \%$ & $13,27 \%$ & $22,94 \%$ & $18,61 \%$ & 13,29 \\
\hline & & I & $37,24 \%$ & $25,93 \%$ & $22,84 \%$ & $14,00 \%$ & 12 & $38,70 \%$ & $21,30 \%$ & $17,94 \%$ & $22,07 \%$ & 11,25 \\
\hline & \multirow{2}{*}{ А.CT-79-EH } & $\mathrm{E}$ & $41,68 \%$ & $13,60 \%$ & $22,15 \%$ & $22,57 \%$ & 14,56 & $39,65 \%$ & $11,16 \%$ & $20,12 \%$ & $29,07 \%$ & 14,80 \\
\hline & & I & $31,01 \%$ & $23,04 \%$ & $17,99 \%$ & $27,95 \%$ & 11,74 & $31,53 \%$ & $18,66 \%$ & $15,80 \%$ & $34,02 \%$ & 12,73 \\
\hline & \multirow{2}{*}{ D.CT-79-EH } & $\mathrm{E}$ & $36,53 \%$ & $15,04 \%$ & $22,99 \%$ & $25,44 \%$ & 24,04 & $39,31 \%$ & $11,52 \%$ & $19,14 \%$ & $30,03 \%$ & 22,25 \\
\hline & & I & $22,04 \%$ & $27,88 \%$ & $15,64 \%$ & $34,45 \%$ & 17,74 & $32,02 \%$ & $20,14 \%$ & $11,13 \%$ & $36,72 \%$ & 18,37 \\
\hline
\end{tabular}

D.CT-79-EH, las mínimas inversiones habría que realizarlas en cubiertas y suelos en función del tipo de actuación, exterior-suelos, interior-cubiertas.

Si se analiza la amortización teórica en la opción básica se observa que el Pay-back puede ir desde los 10, 12 años para la vivienda entre medianeras A.CT-79-EF con actuación interior a 24,10 años para la edificación aislada D.CT-79-EH con actuación exterior. Para la opción «plus» el modelo que ofrece un mayor retorno de la inversión es la vivienda entre medianeras D.CT-79-EH, y la vivienda aislada A.CT-79-EF la que ofrece un menor periodo.

Los períodos de retorno de ambos tipos de rehabilitaciones (básica-plus) son sensiblemente parecidas, tal indicador desvela que teóricamente el sobrecoste de la opción plus se ve suplido por el ahorro energético que conllevan tales incrementos en las mejoras térmicas de la envolvente, como puede verse en la Tabla 5, y, por tanto, a largo plazo es considerablemente mejor que la opción «plus», aunque su desembolso inicial sea mucho mayor.

\section{CONCLUSIONES}

Mediante la implementación de una herramienta generada a partir de las normas UNE-EN ISO 13.790:2011 (20) y ASTM E917-05 2013 se han generado indicadores económicos validos para la evaluación de mejoras en viviendas existentes en función de distintas casuísticas, aportando conocimientos económicos para la ejecución de mejoras energéticas.
Cuando se actúa en viviendas construidas bajo estándares del CT-79, el nivel de mejoramiento deberá de ser mayor para alcanzar ahorros que permitan amortizar la inversión, alcanzándose una diferencia entre una rehabilitación básica o "plus» de entorno a un $20 \%$ en los ahorros para cualquier tipología. Sin embargo, para viviendas con estructura de fábrica la diferencia de aplicar ambos tipos de rehabilitaciones supone un incremento en el ahorro de entre 12 y $14 \%$, siendo menor en función de su vinculación a otros edificios medianeros.

A la hora de estudiar los muros hay que tener en cuenta que la inversión en un edificio aislado A.CT-79-EH actuando exteriormente es tres veces superior a la de una vivienda entre medianeras D.CT-79-EH con actuación interior. A mayor grado de rehabilitación menor será la diferencia entre ambas. Cuanto mayor sea el grado de mejoramiento en los muros menor será la influencia del tipo de intervención (exterior o interior), la normativa con la cual se ejecutó/sistemas constructivos, la relación con otras edificaciones (aislada, pareada o entre medianeras).

En suelos se puede decir que la inversión en un edificio A.CT79-EH actuando exteriormente es un $27 \%$ inferior a la actuación interior, a mayor grado de rehabilitación mayor será la diferencia entre ambas. Cuanto mejor sea el grado de mejoramiento mayor será la influencia del tipo de intervención (exterior o interior), no influyendo de forma determinante la normativa con la cual se ejecutó/sistemas constructivos o la relación con otras edificaciones (aislada, pareada o entre medianeras). 
En la asignación de recursos económicos para cubiertas habrá que tener en cuenta que la inversión en un edificio A.CT79-EH mejorando exteriormente es un $54 \%$ superior a la de una vivienda entre D.CT-79-EH con actuación interior, con un mayor grado de rehabilitación no se producen diferencias sustanciales entre ambas; sin embargo, mayor será la influencia de la elección del tipo de actuación.

Los mejoramientos en carpinterías a través de una opción básica son entre un 39 y un $53 \%$ menor que la opción «plus», en función del número de huecos a rehabilitar. El modelo de estudio para edificios A.CT-79-EF se simuló con carpinterías de madera, por ello a la hora de alcanzar un ni- vel básico solo habría que rehabilitar los huecos orientados hacia el norte, lo que conlleva una diferencia en la opción básica un $43 \%$ con respecto al resto de los casos y un $56 \%$ en la opción plus.

Los mayores períodos de retorno serán para edificaciones posteriores a la entrada en vigor del CT-79 con actuación exterior, tal valor desde un estudio teórico de amortizaciones no parece ser determinante para decantarse por una opción básica o plus puesto que sus valores son sensiblemente cercanos, si bien es cierto que a largo plazo, es considerablemente mejor que la opción plus, aunque su desembolso inicial sea mucho mayor.

\section{REFERENCIAS}

(1) Unión Europea. (2003, 4 de enero). Directiva 2002/91/CE del parlamento europeo y del consejo de 16 de diciembre de 2002 relativa a la eficiencia energética de los edificios. Diario Oficial de la Unión Europea, no 001, pp. 65-71.

(2) Unión Europea. (2010, 18 de junio). Directiva 2010/31/UE del Parlamento Europeo y del Consejo de 19 de mayo de 2010, relativa a la eficiencia energética de los edificios. Diario Oficial de la Unión Europea, n⿳0 153/13.

(3) Unión Europea (2012, 14 de noviembre). Directiva 2012/27/UE del Parlamento Europeo y del Consejo, de 25 de octubre de 2012, relativa a la eficiencia energética, por la que se modifican las Directivas 2009/125/CE y 2010/30/UE, y por la que se derogan las Directivas 2004/8/CE y 2006/32/CE Texto pertinente a efectos del EEE. Diario Oficial de la Unión Europea, $\mathrm{n}^{0} 315$, pp. 1-56.

(4) IDAE. (2011). Proyecto SECH-SPAHOUSE - Análisis del consumo energético del sector residencial en España, p. 7. Instituto para la Diversificación y el Ahorro de la Energía (IDAE) - Secretaría General, Dpto. de Planificación y Estudios.

(5) IDAE. (2011). Plan de ahorro y eficiencia energética 2011-202O, p. 203. Instituto para la Diversificación y el Ahorro de la Energía (IDAE).

(6) IDAE. (2014). Plan de ahorro y eficiencia energética 2014-2020, p. 91. Instituto para la Diversificación y el Ahorro de la Energía (IDAE).

(7) Escorcia, O., García, R., Trebilcock, M., Celis, F., Bruscato, U. (2012). Mejoramientos de envolvente para la eficiencia energética de viviendas en el centro-sur de Chile. Informes de la Construcción, 64(528): 563-574, doi: http://dx.doi. org/10.3989/ic.11.143.

(8) Phil, A. G., Niall, K. (2015). The impacts of household retrofit and domestic energy efficiency schemes: A large scale, ex post evaluation. Energy Policy, 84: 35-43, doi: http://dx.doi.org/10.1016/j.enpol.2015.04.020.

(9) Charlot-Valdieu, C., Outrequin, P. (2011). An approach and a tool for setting sustainable energy retrofitting strategies referring to the 2010 EPBD. Informes de la Construcción, 63(Extra): 51-60, doi: http://dx.doi.org/10.3989/ic.11.064.

(10) Unión Europea. (2012, 21 de marzo). Reglamento Delegado (UE) n ${ }^{0}$ 244/2012 de la Comisión, de 16 de enero de 2012, que complementa la Directiva 2010/31/UE del Parlamento Europeo y del Consejo, relativa a la eficiencia energética de los edificios, estableciendo un marco metodológico comparativo para calcular los niveles óptimos de rentabilidad de los requisitos mínimos de eficiencia energética de los edificios y de sus elementos. Diario Oficial de la Unión Europea, ${ }^{\circ}$ 81, pp. 18-36.

(11) Unión Europea (2012, 19 de abril). Directrices que acompañan al Reglamento Delegado (UE) no 244/2012 de la Comisión, de 16 de enero de 2012, que complementa la Directiva 2010/31/UE del Parlamento Europeo y del Consejo, relativa a la eficiencia energética de los edificios, estableciendo un marco metodológico comparativo para calcular los niveles óptimos de rentabilidad de los requisitos mínimos de eficiencia energética de los edificios y de sus elementos: 2012/C/ 115/o1. Diario Oficial de la Unión Europea, C 115/1.

(12) Vega Catalán, L. (2010, 4 de octubre). La rehabilitación en el Código Técnico de la Rehabilitación. En Congreso Internacional, Rehabilitación y Sostenibilidad. El futuro es posible. Barcelona.

(13) Trebilcock, M. (2011). Percepción de barreras a la incorporación de criterios de eficiencia energética en las edificaciones. Revista de la construcción, 10(1): 4-14, doi: http://dx.doi.org/10.4067/So718-915X2011000100002.

(14) Celis, F., Escorcia, O, Díaz, M., García Alvarado, R., Echeverría, E. (2012). Incidencia de la forma y envolvente en el desempeño térmico de las viviendas del cetro-sur de Chile. Hábitat Sustentable, 2(2): 19-33.

(15) García-Navarro, J., González-Díaz, M. J., Valdivieso, M. (2014). «Estudio Precost\&e»: evaluación de los costes constructivos y consumos energéticos derivados de la calificación energética en un edificio de viviendas situado en Madrid. Informes de la Construcción, 66(535): e026, doi: http://dx.doi.org/10.3989/ic.13.052.

(16) Ruá, M.J., López-Mesa, B. (2012). Spanish energy rating labelling of buildings and its cost implications. Informes de la Construcción, 64(527): 307-318, doi: http://dx.doi.org/10.3989/ic.11.028.

(17) Ahern, C., Griffiths, Ph., O’Flaherty, M. (2013). State of the Irish housing stock-Modelling the heat losses of Ireland's existing detached rural housing stock \& estimating the benefit of thermal retrofit measures on this stock. Energy Policy, 55: 139-151, doi: http://dx.doi.org/10.1016/j.enpol.2012.11.039.

(18) Ministerio de Fomento. (2013, 12 de septiembre). Orden FOM /1635/2013 del 10 de septiembre por el que se actualiza el Documento Básico DB-HE. Boletín Oficial del Estado, nº 219, pp: 67137-67209.

(19) Ramírez de Arellano, A. (2014). Presupuestación de obras. Sevilla: Universidad de Sevilla. 
(20) AENOR. (2011). UNE-EN ISO 13790:2011 Eficiencia energética de los edificios. Cálculo del consumo de energía para calefacción y refrigeración de espacios. Asociación Española de Normalización (AENOR).

(21) ASTM. (2013). ASTM E917-05-13: Standard Practice for Measuring Life-Cycle Costs of Buildings and Building Systems. ASTM International.

(22) CTE-Ministerio de Fomento. (2014). Documento de Apoyo al Documento Básico DB-HE Ahorro de energía. DA DBHE/3 Puentes Térmicos. Código Técnico de la Edificación-Ministerio de Fomento.

(23) IDAE. (2014). Factores de emisión de CO2 y coeficientes de paso a energía primaria de diferentes fuentes de energía final consumidas en el sector edificios en España. http://www.minetur.gob.es/energia/desarrollo/EficienciaEnergetica/ RITE/propuestas/Documents/2014_03_03_Factores_de_emision_CO2_y_Factores_de_paso_Efinal_Eprimaria_V. pdf. Instituto para la Diversificación y el Ahorro de la Energía (IDAE).

(24) Eurostat Stadistics Explainted. Electricity and natural gas price statistics. http://ec.europa.eu/eurostat/statisticsexplained/index.php/Electricity_and_natural_gas_price_statistics.

(25) Luxan García de Diego, M., Gómez Muñoz, G., Román López, E. (2015). Cuentas energéticas no habituales en edificación residencial. Informes de la Construcción, 67(Extra-1): mo28, doi: http://dx.doi.org/10.3989/ic.14.059. 